OPEN

SUBJECT AREAS:

CELLULAR

NEUROSCIENCE

NEUROTROPHIC FACTORS

NEUROCHEMISTRY

CELL GROWTH

Received

5 February 2013

Accepted

23 July 2013

Published

9 August 2013

Correspondence and requests for materials should be addressed to J.R.-A. (jose. rodriguez@uab.es)

\section{X-linked Inhibitor of Apoptosis Protein} negatively regulates neuronal \section{differentiation through interaction with CRAF and Trk}

\author{
Rut Fadó1,3, Rana S. Moubarak ${ }^{2,3}$, Alfredo J. Miñano-Molina ${ }^{1,3}$, Bruna Barneda-Zahonero ${ }^{1,3}$, \\ Jorge Valero ${ }^{1,3}$, Carlos A. Saura ${ }^{1,3}$, Julio Moran ${ }^{4}$, Joan X. Comella ${ }^{2,3}$ \& José Rodríguez-Álvarez ${ }^{1,3}$
}

${ }^{1}$ Institut de Neurociències and Dpt. Bioquímica and Biología Molecular, Universitat Autònoma de Barcelona, 08193 Cerdanyola del Vallès, Spain, ${ }^{2}$ Institut de Recerca de I'Hospital Universitari de la Vall d'Hebron, 08035 Barcelona, Spain, ${ }^{3}$ Centro de Investigación Biomédica en Red sobre Enfermedades Neurodegenerativas (CIBERNED), Spain, ${ }^{4}$ División de Neurociencias, Instituto de Fisiología Celular, Universidad Nacional Autónoma de México, 04510 México D.F., México.

X-linked Inhibitor of apoptosis protein (XIAP) has been classically identified as a cell death regulator. Here, we demonstrate a novel function of XIAP as a regulator of neurite outgrowth in neuronal cells. In PC12 cells, XIAP overexpression prevents NGF-induced neuronal differentiation, whereas NGF treatment induces a reduction of endogenous XIAP levels concomitant with the induction of neuronal differentiation. Accordingly, downregulation of endogenous XIAP protein levels strongly increases neurite outgrowth in PC12 cells as well as axonal and dendritic length in primary cortical neurons. The effects of XIAP are mediated by the mitogen-activated protein kinase (MEK)/extracellular signal-regulated kinases (ERKs) pathway since blocking this pathway completely prevents the neuritogenesis mediated by XIAP downregulation. In addition, we found that XIAP binds to $\mathrm{cRaf}$ and Trk receptors. Our results demonstrate that XIAP plays a new role as a negative regulator of neurotrophin-induced neurite outgrowth and neuronal differentiation in developing neurons.

nhibitor of apoptosis proteins (IAPs) are a conserved family of proteins characterized by the presence of at least one Baculoviral IAP Repeat (BIR) domain ${ }^{1}$ and are present in a wide range of organisms. Most IAPs display anti-apoptotic properties and some can directly inhibit the activity of caspases ${ }^{2}$. However, recent studies have demonstrated the importance of IAPs in various cellular processes, including cell proliferation, migration, motility, immune response and intracellular signalling ${ }^{3-5}$.

XIAP is the best-studied member of the IAP family in mammals. It has three BIR domains at the amino terminus and a Ubiquitin Binding Domain (UBA) and a RING domain with an E3 ubiquitin ligase activity at the carboxy terminus 6 . XIAP-mediated inhibition of caspases is mediated by their interaction through the BIR1-BIR2 linked region and a surface groove in BIR3 domain $^{7-9}$. On the other hand, the RING domain mediates XIAP ubiquitination and trans-ubiquitination of interacting proteins such as other IAP family members, caspases- 3 and $-9^{10-12}$ and proteins implicated in the activation of signalling cascades, including JNK, TGF $\beta, N F \kappa B, A k t$, and MEK pathways ${ }^{13-17}$.

Studies with XIAP-deficient mice have revealed the importance of this protein in proliferation and differentiation processes. For instance, XIAP is involved in mammary gland development, T lymphocytes maturation and ventral mesoderm differentiation ${ }^{17-19}$. Although, the specific role of XIAP in neuronal differentiation and development has not been addressed, some XIAP interacting proteins have been shown to regulate these pro$\operatorname{cesses}^{20}$. Moreover, several studies have recently shown that anti-apoptotic proteins are also able to modulate neurite growth and neuronal differentiation. Bcl-2, FAIM-S and FLIP modulate neuronal growth in different neuronal types through the regulation of the Trk/MEK/ERK pathway ${ }^{21-23}$, suggesting that XIAP could have an analogous function.

In the present study, we show for the first time a new biological function of XIAP, which acts as an endogenous modulator of neurite growth during neuronal differentiation. We show that NGF induces a decrease in XIAP levels in PC12 cells that is concomitant with neurite outgrowth. In the same sense, XIAP overexpression blocks 
NGF-mediated neuritogenesis in PC12 cells whereas downregulation of endogenous XIAP increases neurite outgrowth in PC12 and axonal and dendritic length in cultured cortical cultures. XIAPdependent regulation of neuritogenesis is mediated by the activation of the MEK/ERK pathway through the interaction of XIAP with cRaf and Trk receptors.

\section{Results}

XIAP is downregulated during neuronal differentiation. In order to explore whether XIAP is regulated during neuronal differentiation, we decided to analyse XIAP protein levels in rat brain at different embryonic and adult stages. Biochemical analyses revealed high levels of XIAP in cerebral cortex at embryonic stage E17 and a decrease at postnatal stages . At postnatal day 4, the levels are half than those at E17 and remain constant until adulthood. The decrease of XIAP levels in the cerebral cortex with aging is also evident in cultured cortical neurons. During the first days in culture, XIAP levels remain constant then decrease starting at 4 DIV (Fig. S1).

In order to determine whether the observed changes in XIAP expression are related to neurotrophin-mediated neuronal differentiation, we assessed NGF-dependent neurite outgrowth in PC12 cells. As shown in Figure S1C, NGF induced a decrease in XIAP protein levels when compared with vehicle-treated cells (significant at 20 hours; $\mathrm{p}<0.01$ ). NGF-induced downregulation of XIAP is dependent on the MEK/ERK pathway activity since it can be prevented with MEK inhibitors such as PD98059 or U0126 (Fig. S1D). The efficiency of PD98059 and U0126 to inhibit the MEK/ERK pathway was verified by the observed decrease of ERK phosphorylation (Fig. S1D).

XIAP regulates NGF-dependent neurite outgrowth. We next investigated whether NGF-mediated decrease in XIAP levels is related with neuritogenesis by analysing the effects of gain or loss of function through overexpression or knock-down of XIAP. PC12 cells overexpressing XIAP showed a marked decrease in NGFmediated neurite outgrowth (Fig. 1A). Only half of the cells that overexpressed XIAP developed neurites 48 hours after NGF stimulation as compared with control-transfected cells (Fig. 1B). The expression of myc-tagged human XIAP was verified by immunoblotting (Fig. 1C). By contrast, opposite results were observed when XIAP expression was downregulated by specific shRNA. PC12 cells were infected with XIAP shRNA encoding vectors or the scrambled sequence and were cultured for 3 days before NGF a
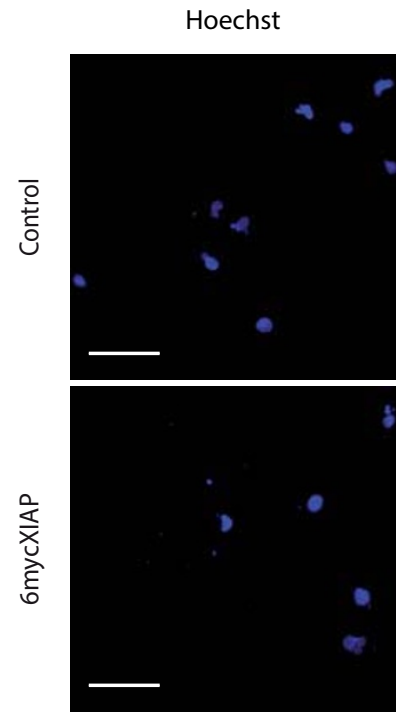

b

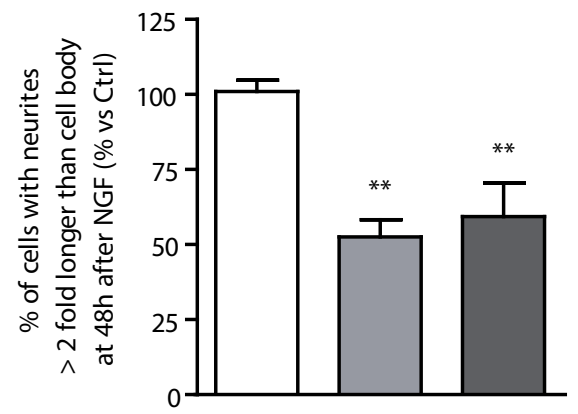

Control

6mycXIAP

XIAP-I-GFP

GFP/ cMyc
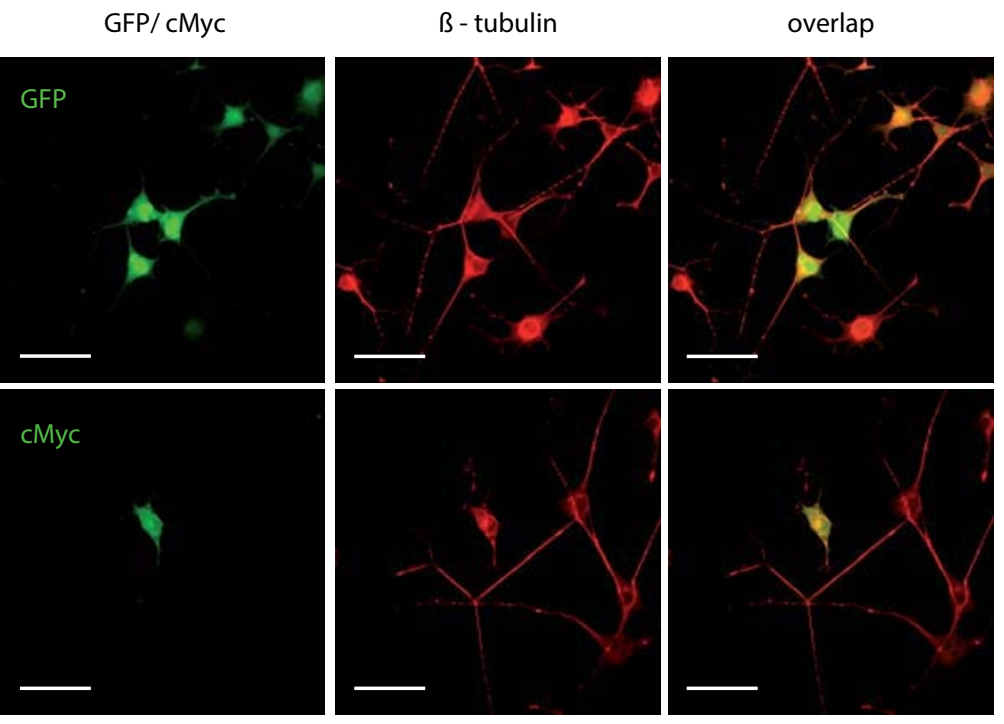

C

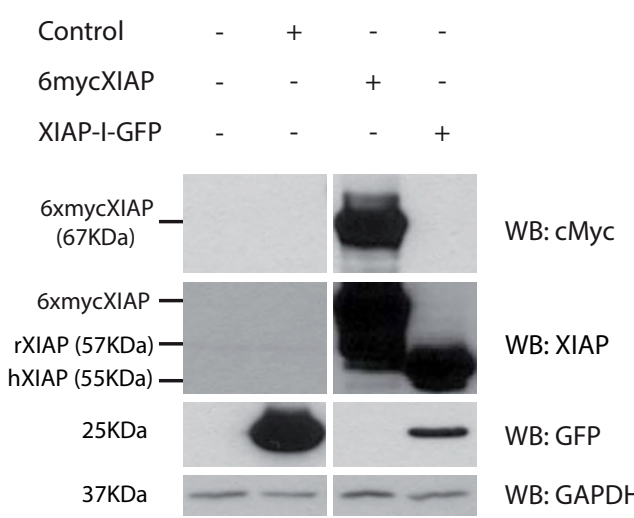

Figure 1 XIAP overexpression reduces NGF-induced neurite outgrowth. PC12 cells cultured during 24 hours were transfected with overexpressing XIAP vectors (pcDNA3-6mycXIAP or pBMN-XIAP-I-GFP) or with a control vector (pEGFP). Two days later, cells were serum deprived for 6 hours and treated with NGF for 48 hours. Myc positive cells were detected by immunociytochemistry, while $\beta$-tubulin and Hoechst were used to stain neurites and nucleus respectively. (a) Representative photographs are shown. (b) Results are given as percentage of Myc or GFP positive cells with neurites $>2$ fold longer than the cell body. Values are the mean \pm SEM of 3 independent experiments performed in duplicate. ${ }^{* *}$ p $<0.01$ vs. control. (c) Overexpressed XIAP was detected by western blotting. The gel images were cropped around the region of interest and the samples were resolved in the same gel. 
a

shscrambled RNAi

ShXIAP RNAi_1

$57 \mathrm{KDa}$

$34 \mathrm{KDa}$

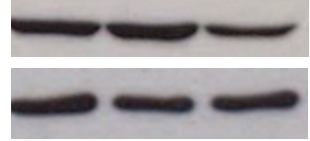

XIAP

GAPDH

C

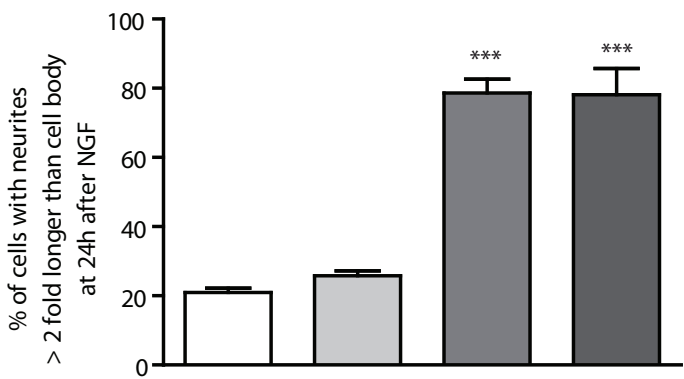

shscrambled RNA

shXIAP RNAi_1

ShXIAP RNAi_2

e

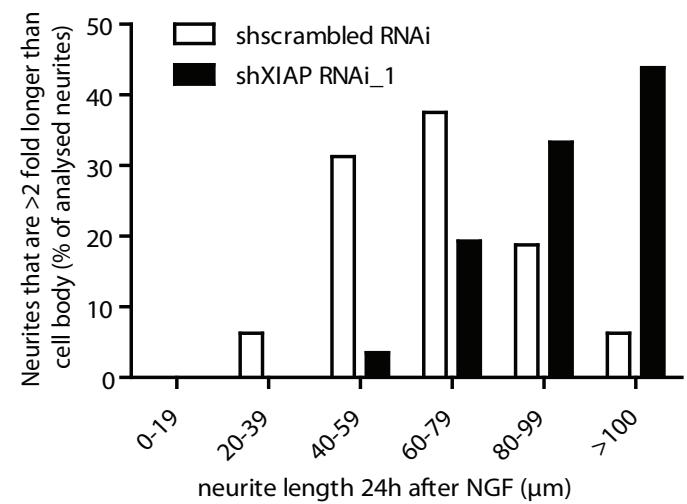

g

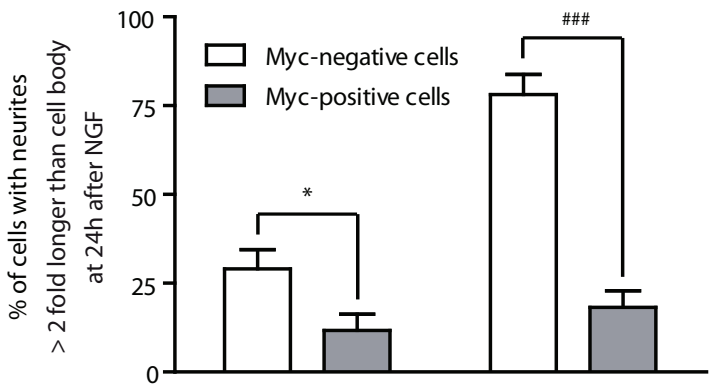

shscrambled RNAi $\quad+\quad+$

ShXIAP RNAi

b

shscrambled RNAi_1

shscrambled RNAi_2

shXIAP RNAi_1

shXIAPRNAi_2

xiap

$18 s$

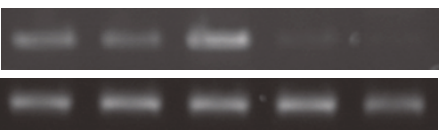

d

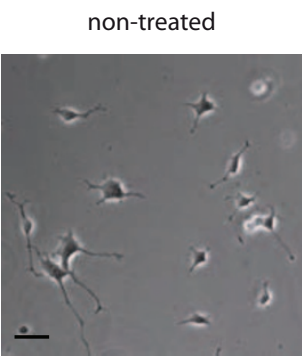

ShXIAP RNAi_1

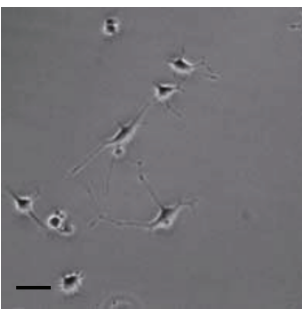

shscrambled RNAi
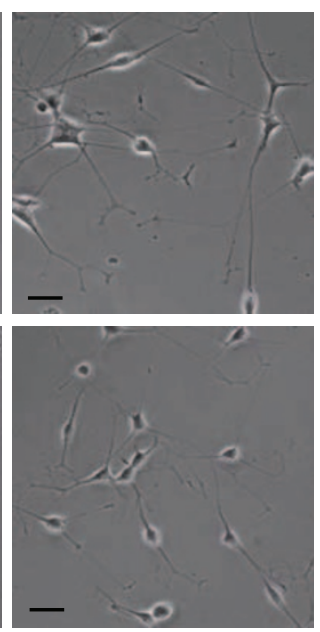

ShXIAP RNAi_2

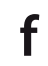

phase contrast
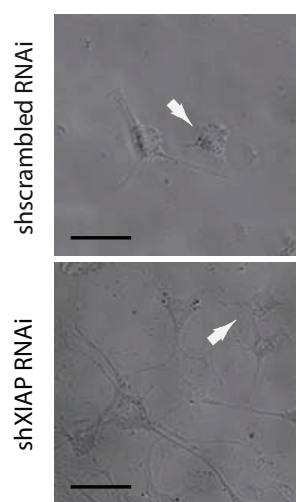

Tag: GFP
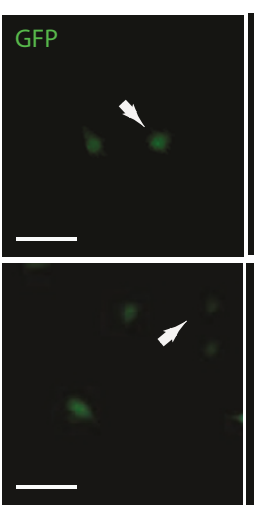

Tag: cMyc

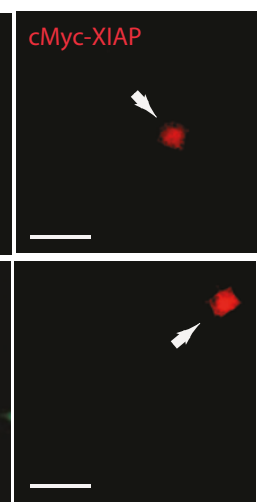

Figure $2 \mid$ XIAP down regulation accelerates NGF-induced neurite outgrowth. (a) PC12 cells were infected with lentiviral shXIAP $\left(1 \times 10^{6}\right.$ TU/ml). XIAP protein levels were determined at 3DIV by western blotting. GAPDH protein levels were used as loading control. The gel images were cropped around the region of interest and the samples were resolved in the same gel. (b) PC12 cells were infected with lentiviral RNA interference for XIAP with two independent RNAi sequences against XIAP (RNAi_1 and RNAi_2). XIAP mRNA levels were determined by RT-PCR. 18 s mRNA levels were used as loading control. (c-d) Neurite outgrowth was analysed in PC12 cells infected with two independent RNAi sequences against XIAP 24 h after NGF addition. Results are shown as in a. (e) Characterization of NGF-mediated neurite outgrowth in shXIAP treated cells. Only neurites $>2$ fold longer than the cell body emerging from the soma were analysed. Histogram shows the percentage of analysed neurites that have the indicated length. ${ }^{*} \mathrm{p}<0.05$ and $* * * \mathrm{p}<0.001$ vs. shscrambled; ( $\mathrm{f}-\mathrm{g}$ ) XIAP overexpression blocks shXIAP-induced neurite outgrowth. PC12 cells were infected at 0 DIV and transfected at $1 D I V$ as previously explained in the methods section. At 3DIV, cells were serum deprived during 6 hours and NGF was added for 24 hours. XIAP overexpressing cells were detected by immunocytochemistry against Myc. GFP signal indicated XIAP downregulated cells. Representative photographs are shown in a and neuritic growth results are given in $b$ as percentage of GFP positive cells with neurites $>2$ fold longer than the cell body. Values are the mean \pm SEM of 3 independent experiments performed in duplicate. ${ }^{*} \mathrm{p}<0.05$ vs. scrambled Myc-negative; ${ }^{\# \#} \mathrm{p}<0.001$ vs. shXIAP Myc-negative. 
stimulation. Efficiency of XIAP downregulation was confirmed at the protein level (Fig. 2A) or at the mRNA level with two independent sequences of shRNA (shXIAP RNAi_1 and shXIAP RNAi_2: Fig. 2B). Neurite outgrowth is increased as soon as $24 \mathrm{~h}$ after NGF stimulation $(80.3 \pm 2.6 \%$ shXIAP RNAi_1 vs. $27.4 \pm 2.0 \%$ shscrambled RNAi; Fig. 2C and D). In addition, a higher frequency of longer neurites was observed in shXIAP RNAi-infected cells than in shscrambled RNAi-infected cells (Fig 2E). In contrast, no changes in the number of branching points per cell were observed in shXIAP RNAi-infected cells compared with control cells ( $1.29 \pm 0.31$ vs. 1.05 $\pm 0.23)$. The effect of shXIAP RNAi on neurite outgrowth is due to a specific downregulation of endogenous XIAP levels, because it can be prevented when XIAP-RNAi infected cells were simultaneously transfected with human XIAP which is not sensible to rat shXIAP RNAi (Fig. 2F and G). Altogether, these results demonstrate that XIAP overexpression impairs NGF-induced neurite outgrowth while its downregulation causes the opposite effect.

Endogenous XIAP downregulation induces neurite outgrowth in the absence of NGF in PC12 cells. Our results so far demonstrated that NGF-mediated neurite outgrowth was enhanced by decreasing XIAP levels and reduced by XIAP overexpression. In order to investigate whether reduction of XIAP levels would trigger neurite outgrowth independently of NGF, we analysed the effect of XIAP downregulation in PC12 cells, in the absence of neurotrophic stimulation. As shown in Figure 3A, XIAP downregulation induced neurite formation in PC12 cells in the absence of NGF. Moreover, cellular proliferation was reduced (Fig S2A) and progression into the cell cycle stopped by XIAP downregulation (Fig. S2B). Moreover, shXIAP-induced enhancement of neurite length was related to the lower cell number in shXIAP cultures since neurite formation was not related with culture cell density (Fig. S3). Neurite outgrowth mediated by XIAP downregulation was dependent on shRNA concentration (viral titration; Fig. 3B) and was observed with two different shXIAP RNAis $(30.6 \pm 5.2$ vs. $22.9 \pm 5.7 \%$ for RNAi_1 and RNAi_2, respectively; Fig. 3C). The absence of morphological changes in control PC12 cells infected with lentivirus was corroborated using two additional scrambled sequences (data not shown). A detailed characterization of the number of cells with neurites 2 fold longer than the diameter of the cell body revealed that PC12 cells infected with shXIAP RNAi displayed an increase in the percentage of cells that presented more than 1 neurite (3\% in control vs. $49 \%$ in shXIAP; Fig. 3D). A higher frequency of longer neurites (Fig. 3E) as well as a higher average of neurite length $(71.1 \pm 3.8$ vs. $32.2 \pm 1.5 \mu \mathrm{m} ; \mathrm{p}<0,001)$ was observed in the shXIAP RNAi infected vs.control cells.

Downregulation of XIAP increases axonal and dendritic length in cortical neurons. Next, we analysed the role of XIAP in neurite morphogenesis in primary cortical neurons by using XIAP shRNA. Efficiency of XIAP downregulation was confirmed by RT-PCR and immunocytochemistry (Fig. 4A-B). shXIAP RNA increased neurite length in cortical neurons at 4 DIV, compared with control cells (Fig. 4C-D). Axonal measurements were performed by immunocytochemistry with anti-SMI 312, a pan-axonal neurofilament marker, whereas dendrites were labelled with anti-microtubule-associated protein 2 (MAP-2). Our results showed that downregulation of XIAP in the absence of neurotrophin stimulation, causes an increase in axonal length (Fig. 4E; the average length in shXIAP RNAi infected cells was $92.1 \pm 0.8$ vs. $72.5 \pm 1.6 \mu \mathrm{m}$ in control; $\mathrm{p}<0.01$ ) and dendritic length (Fig. 4F; the average length in shXIAP RNAi cells was $32.8 \pm 1.6$ vs. $25.8 \pm 1.9 \mu \mathrm{m}$ in control; $\mathrm{p}<0.05)$ in cortical neurons. These results indicate that XIAP negatively regulates neurite growth, both in PC12 cells and in cortical neurons.

MEK/ERK pathway is involved in XIAP-mediated regulation of neurite outgrowth. Sustained activation of MEK/ERK cascade is considered to be the main intracellular pathway involved in NGFmediated neurogenesis ${ }^{24,25}$. To address whether the activation of this signalling pathway is required for neurite formation induced by XIAP downregulation, we analysed the effect of pharmacological inhibition of MEK/ERK pathway. The presence of the MEK inhibitors, PD98059 or U0126, resulted in a significant reduction in neurite outgrowth mediated by XIAP down regulation $(85 \%$ and $74 \%$ inhibition respectively; Fig. 5A). Accordingly, PD98059 also caused a significant reduction in NGF-mediated neurite outgrowth caused by XIAP downregulation(control $77.5 \pm 3.4 \%$ vs. PD98059 $49.0 \pm 4.7 \%$ of cells; Fig. $5 B$ ).Downregulation of XIAP expression in NGF-treated cells resulted in an increase of ERK phosphorylation in the cytosol and nucleus (Fig. 5C). Activation of the MEK/ERK pathway was further validated by an increase in CREB phosphorylation induced by XIAP downregulation (Fig. 5D), an ERKactivated transcription factor that triggers neuritogenesis in $\mathrm{PC}_{2} 2^{26}$. These results suggest a role of MEK/ERK pathway on neurite outgrowth induced by XIAP down regulation.

NGF prevents the binding of XIAP to cRaf. The only well-characterized connection between XIAP and elements of MEK/ERK signalling pathway is the direct interaction with cRaf, also called Raf $1^{16}$. It is also known that binding of NGF to its receptor triggers Raf activation and some Raf isoforms have been previously related to neurite formation ${ }^{27}$. Therefore we explored the possibility of a XIAP and $c$ Raf interaction that could be important for NGF and shXIAP RNA-mediated neuritogenesis. To address this issue, PC12 cells were lysed after being treated for $1 \mathrm{hr}$ with NGF and an immunoprecipitation assay was performed with XIAP antibody. As shown in Figure 6A, endogenous XIAP specifically interacts with cRaf together with CHIP, another protein associated with the XIAP-cRaf complex, in basal conditions. However, cRaf binding to XIAP was impaired in the presence of NGF. It was described that cRaf-XIAP complex induced cRaf ubiquitination, while XIAP dissociation stabilizes $\mathrm{cRaf}^{16}$. As expected, cRaf was polyubiquitinated in basal conditions, while NGF stimulation promoted its deubiquitination, as we showed by immunoprecipitation of ubiquitinated proteins in PC12 cells lysates pre-treated with a proteosome inhibitor (MG132; Fig. 7B). Moreover, overexpression of XIAP increases cRaf ubiquitination (Fig. 6C). However, several evidences suggest that this ubiquitination does not promote cRaf degradation. We found that cRaf protein levels do not increase in the presence of MG132 and the increase in ubiquitinated cRaf by XIAP overexpression did not result in reduced cRaf protein levels (Fig. 6D). Accordingly, cRaf levels were not increased after XIAP down regulation (Fig. 6E). By contrast, down regulation of endogenous cRaf with sh-cRaf (Fig. 6F) blocked shXIAP-mediated enhancement in neurite outgrowth in cells treated with NGF for 24 h (Fig. 6G). Also, down regulation of cRaf significantly blocked NGF-induced neurite outgrowth after $48 \mathrm{~h}$ (Fig. 6H), confirming that cRaf is necessary for neuritogenesis in PC12 cells.

XIAP interacts with TrkA in the absence of neurotrophin stimulation. Activation of the RAF/MEK/ERK pathway induced by NGF is dependent on Trk receptor stimulation ${ }^{28}$. Some reports have shown that anti-apoptotic proteins such as FAIM-S or FLIP are able to interact with Trk receptors ${ }^{22,23}$. Moreover, the recruitment of XIAP to TAK1-TAB1 at the membrane by BMP (Bone Morphogenetic Protein) receptor has been reported ${ }^{17}$, suggesting that XIAP could be found in association with plasma membrane receptors. These findings suggest that XIAP might affect NGF-mediated stimulation of MEK/ERK pathway upstream of cRaf.

In order to analyse the eventual interaction between TrkA and XIAP, we used HEK293T cells co-transfected with HA-tagged TrkA and with myc-tagged XIAP. Two days after transfection, cells were lysed and Trk immunoprecipitation was performed using a pan-Trk antibody. Figure 7A demonstrates that XIAP specifically 
a

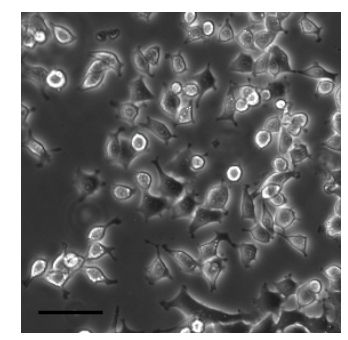

shscrambled RNAi
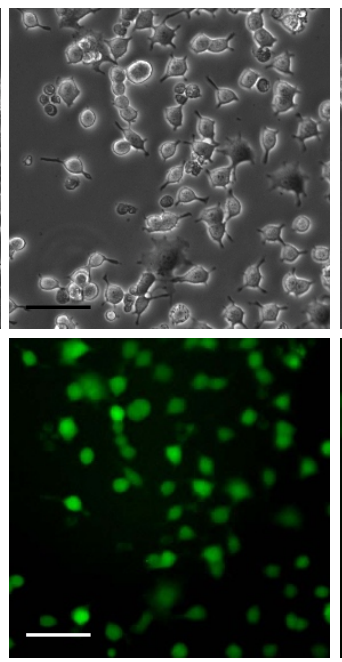

shXIAP RNAi 1
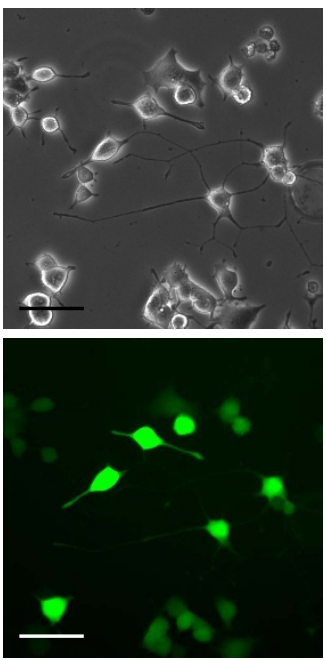

b

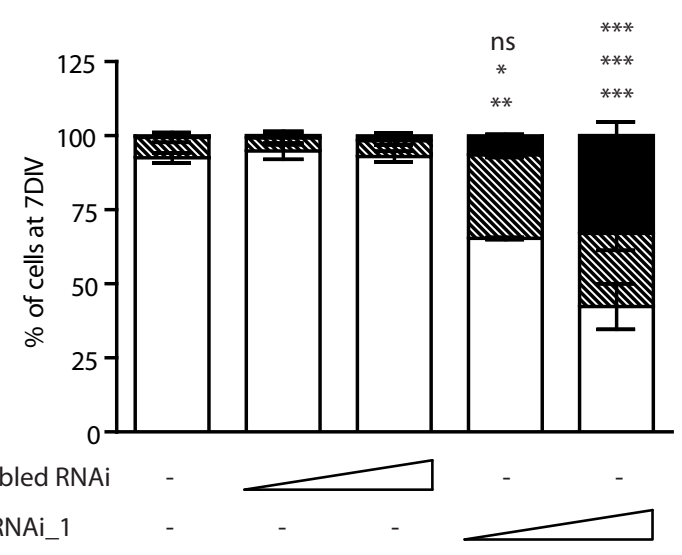

no neurites

neurites 1-2 fold longer than cell body

neurites $>2$ fold longer than cell body

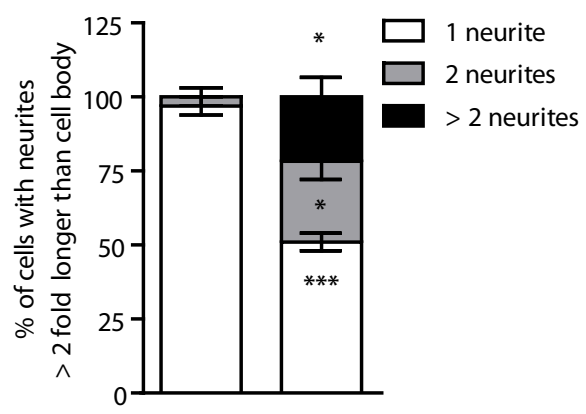

shscrambled RNAi

$+$

C

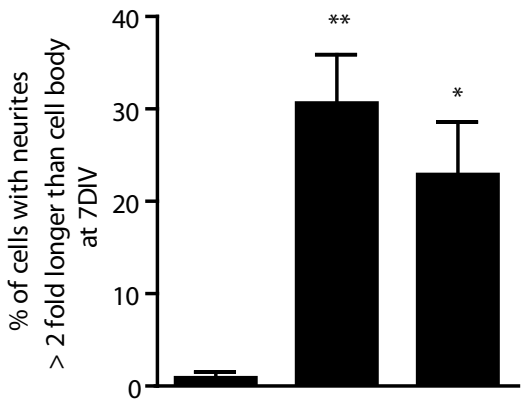

shscrambled RNAi +

shXIAP RNAi_1

shXIAP RNAi_2
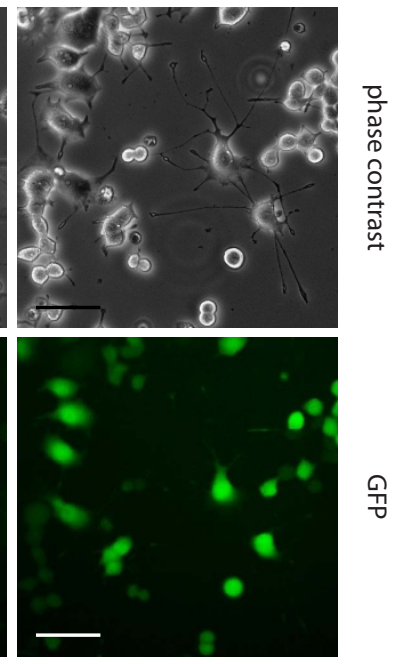

d

shXIAP RNAi_1

shscrambled
shXIAP RNAi_1

\section{(1)}

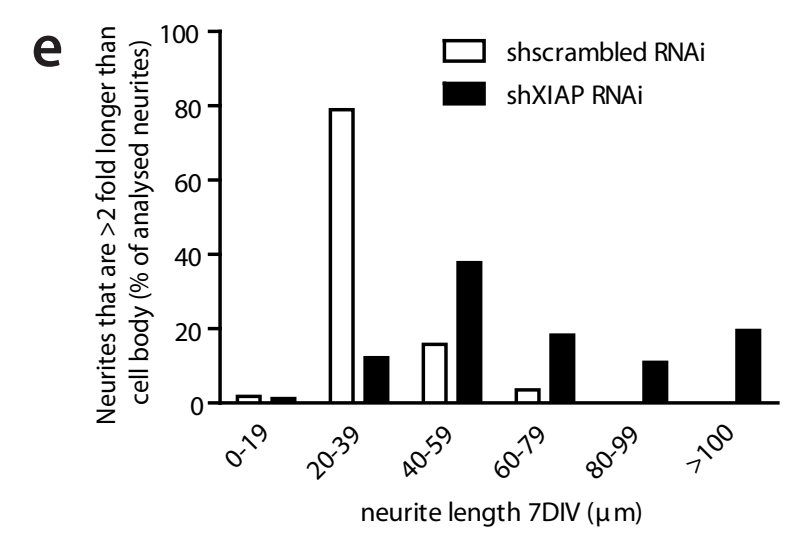

Figure $3 \mid$ Downregulation of XIAP causes neurite outgrowth in PC12 cells. PC12 cells were infected with lentiviral shXIAP at two different titrations ( 1 or $2 \times 10^{6} \mathrm{TU} / \mathrm{ml}$ ) or with two independent RNAi sequences against XIAP (RNAi_1 and RNAi_2). (a) Representative photographs of infected PC12 at 7DIV are shown. (b-c) Neurite outgrowth was determined at 7 days post infection. Results are shown as percentage of cells that present no neurites (white), neurites 1-2 fold longer than cell body (grated) or $>2$ fold longer than cell body (black) and are the mean \pm SEM of 3 independent experiments performed in triplicate. (d-e) Histogram in d shows the percentage of cells at 7 DIV that present 1, 2 or $>2$ neurites, and in e, the percentage of counted neurites that have the indicated length. Only neurites $>2$ fold longer than the cell body emerging from the soma were analysed. One hundred cells infected with shXIAP were monitored and measured per each experiment and pooled by neurite length intervals. Results in (b-d) are the mean \pm SEM of 3 independent experiments performed in triplicate. ${ }^{*} \mathrm{p}<0.05,{ }^{*} \mathrm{p}<0.01,{ }^{* * *} \mathrm{p}<0.001 \mathrm{vs}$. scrambled. Results in e are from pooled cells from 3 independent experiments. 
a

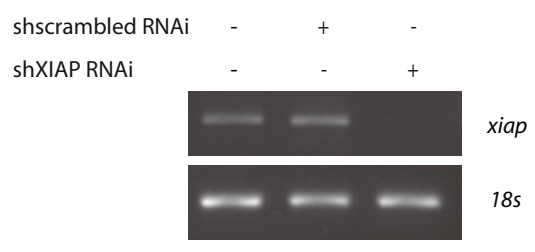

b

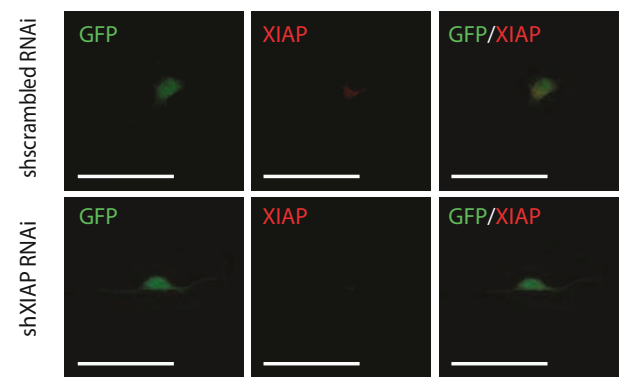

C
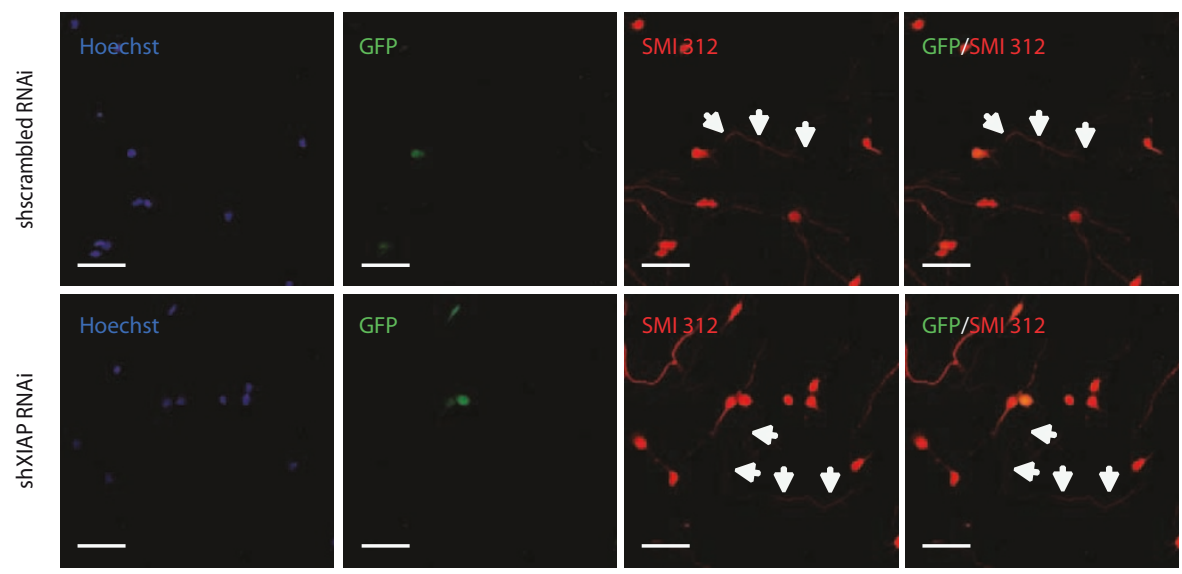

d
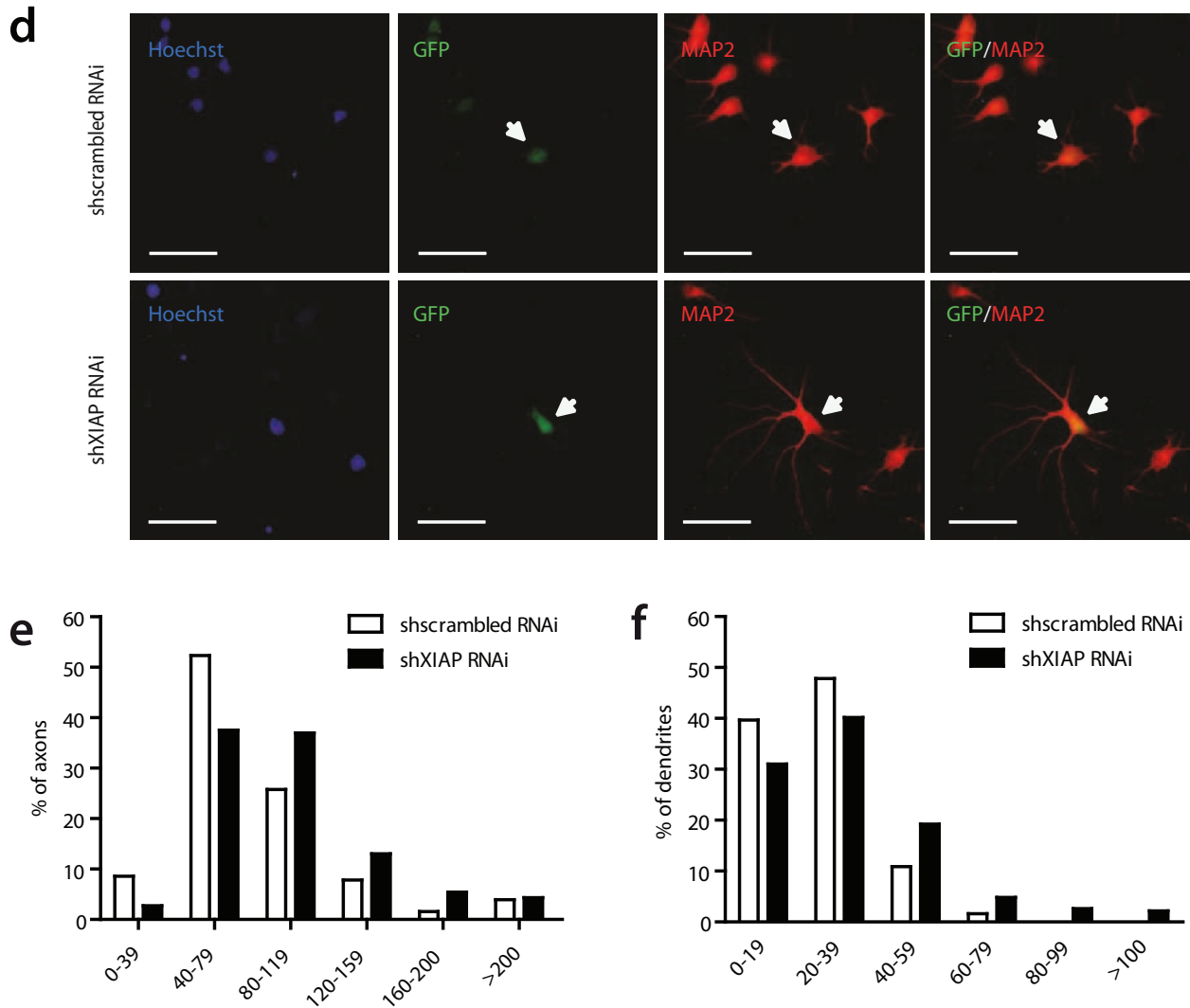

axon length $(\mu \mathrm{m})$

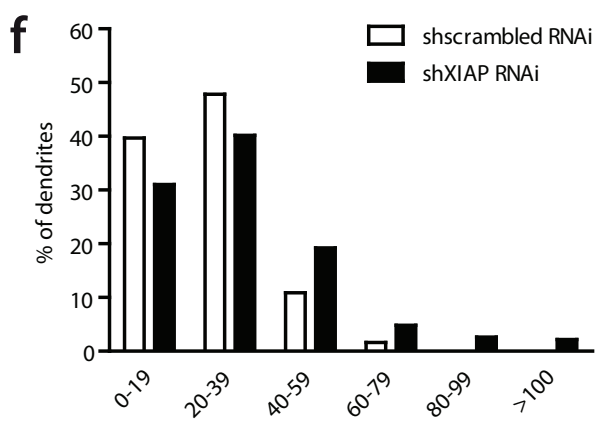

dendrite length ( $\mu \mathrm{m})$

Figure $4 \mid$ Effect of XIAP down regulation on neurite outgrowth of cortical neurons. Cortical cells were infected at 0DIV with sh-scrambled or shXIAPlentivirus and fixed at 4 DIV. (a) Decreased levels of xiap mRNA verified the efficiency of XIAP down regulation in cortical neurons. $18 \mathrm{~s}$ mRNA levels were used as control. The gel images were cropped around the region of interest and the samples were resolved in the same gel.

(b) Immunocytochemistry against XIAP protein confirmed its absence in shXIAP RNAi infected cells. (c) SMI 312 was used as pan-axonal neurofilament marker. (d) MAP2 was used as a neurite marker. (e-f) Measurements of axonal length and dendritic length respectively. Results are the percentage of counted axons or neurites that have the indicated length. Scale bar $=40 \mu \mathrm{M}$. 
a

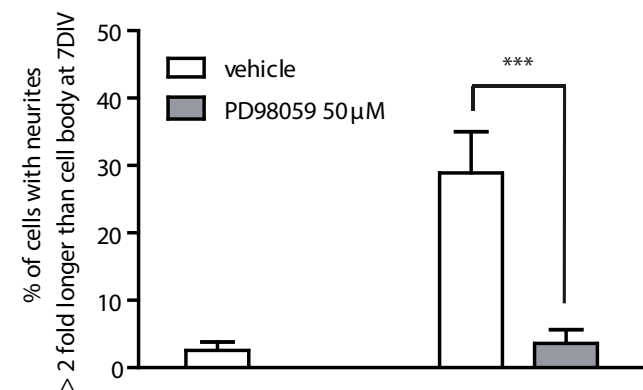

shscrambled RNAi

shXIAP RNAi b

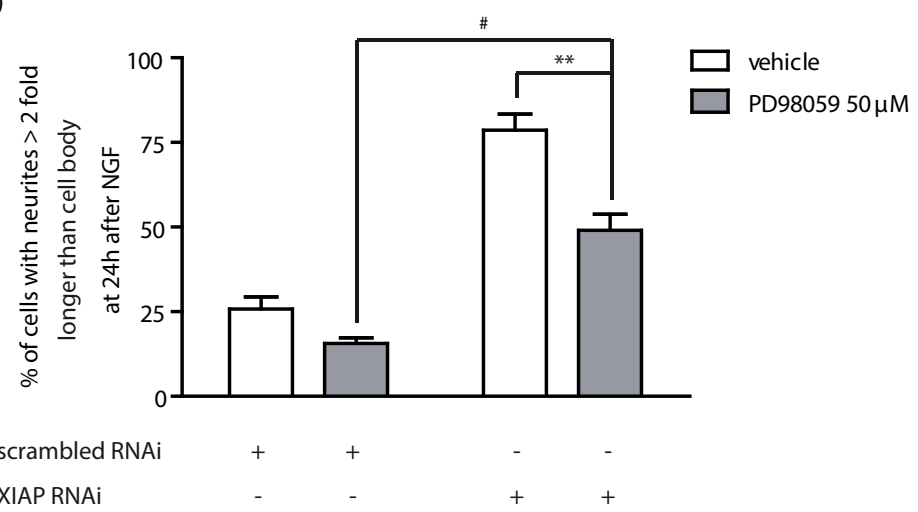

C
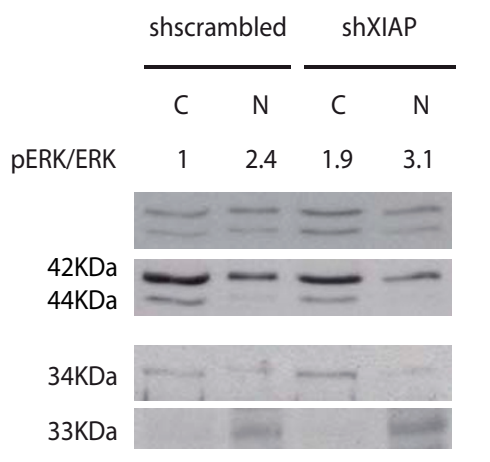

pERK 1

PERK 2

ERK 1

ERK2

GAPDH

Histone $\mathrm{H} 1$ d

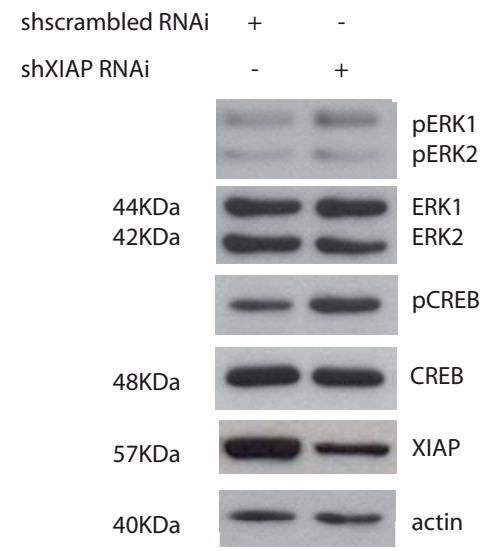

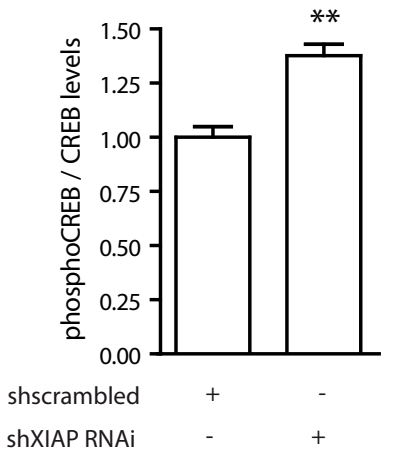

shXIAP RNAi

Figure 5 | Activation of MEK pathway is required for neurite outgrowth induced by XIAP down regulation. (a) MEK inhibition reduces neurite outgrowth induced by shXIAP. PC12 cells were infected at 1 DIV with shXIAP lentivirus and treated with MEK inhibitors (PD98059; $50 \mu$ M, orU0126; $10 \mu \mathrm{M}) 5$ hours after infection. Results are given as percentage of cells at 7DIV with neurites $>2$ fold longer than cell body and are the mean \pm SEM from 3 independent experiments performed in duplicate. ${ }^{* *} \mathrm{p}<0.005$ vs. shXIAP RNAi infected cells. (b) MEK inhibition decreases NGF-induced neuritic outgrowth in shXIAP pre-treated cells. Cells were infected during 3DIV, serum deprived for 6 hours and treated afterwards with NGF for 24 hours. PD98059 (or vehicle) was added 30 minutes before NGF and during the treatment. Results were analysed as in (a). ** $<<0.01$ vs. shXIAP RNAi infected cells; $\# \mathrm{p}<0.01$ vs. shscrambled. (c) Increase in pERK in cytosolic and nuclear fractions of shXIAP RNAi infected cells. Cell lysates were processed as indicated in the methods to obtain the cytosolic (C) and nuclear (N) fractions. pERK and total ERK were determined by western blot as in (a). Histone H1 and GAPDH were used as markers of nuclear and cytosolic fractions respectively. A representative of three western blots is shown. The ratio pERK/ERK was normalised to 1 for cytosolic and nuclear fractions in control cells. (d) XIAP down regulation increases the phosphorylation of CREB. PC12 cells were infected with shXIAP lentivirus as in (a) and phosphoCREB protein levels were detected by WB. The graph represents the ratio between phosphoCREB and total CREB levels. Ratio in control condition was defined as 1 . Values are the mean \pm SEM of 3 independent experiments. $* * p<0.01$ vs. shscrambled. The gel images were cropped around the region of interest and the samples were resolved on gels run under the same experimental conditions.

interacts with TrkA. To validate these results in a neuronal model, PC12 cells were transfected with myc-tagged XIAP and processed as explained above. Again, XIAP interacted with endogenous TrkA in a NGF-independent manner, whereas this interaction was significantly reduced in the presence of NGF (Fig. 7B,C). Finally, additional analyses revealed that endogenous XIAP bound to TrkB, the endogenous BDNF receptor, in embryonic mouse brain lysates (E15; Fig. 7D). These results demonstrate for the first time that XIAP is able to form complexes with Trk receptors.

\section{Discussion}

Although the role of IAPs as anti-apoptotic proteins has been extensively described ${ }^{3}$, they play additional biological functions unrelated to apoptosis modulation. Several reports have demonstrated the interaction of IAPs with new partners ${ }^{15,16}$, adding new perspectives of the cellular roles of IAPs. The present study further contributes to the elucidation of novel roles of IAPs, showing for the first time that XIAP is involved in neuronal differentiation, particularly in neurotrophin-mediated neurite outgrowth through its interaction with cRAF and Trk receptors.

Few data existed until nowadays about the role of XIAP in development or cellular differentiation. XIAP has been related with mammary gland development, $\mathrm{T}$ lymphocytes maturation and ventral mesoderm differentiation ${ }^{17-19}$. Additionally, XIAP was reported to be involved in the control of cell cycle and proliferation in epithelial and endothelial cells and in breast carcinoma cell lines ${ }^{29,30}$. On the other hand, the eventual role of IAPs in the formation of specialised cell structures related to cytoskeleton remodelling is poorly documented. It has been described that the knockdown of XIAP in HeLa cells causes the formation of lamellipodia and filopodia accompanied by enhanced spreading, even in the absence of trophic stimulation ${ }^{16}$. In developing neurons, the formation of lamellipodia and filopodia-like 
A

B

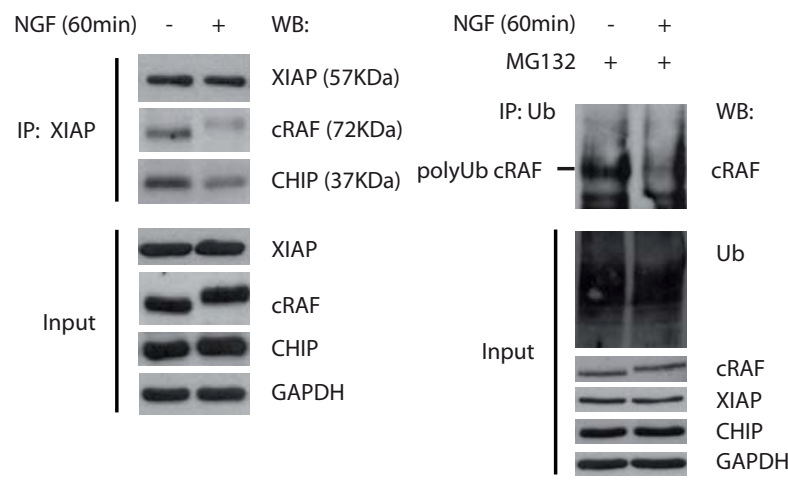

D

$\begin{array}{lllllll}\text { Control } & - & - & + & + & - & \\ \text { 6xmycXIAP } & - & - & - & - & + & + \\ \text { NGF (60min) } & - & + & - & + & - & +\end{array}$

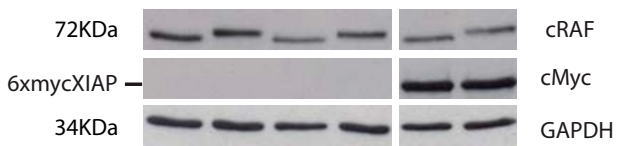

$\mathbf{F}$

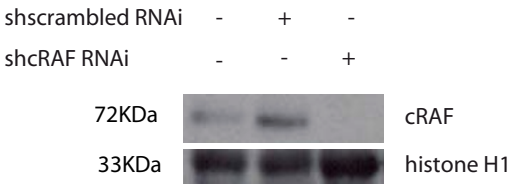

G

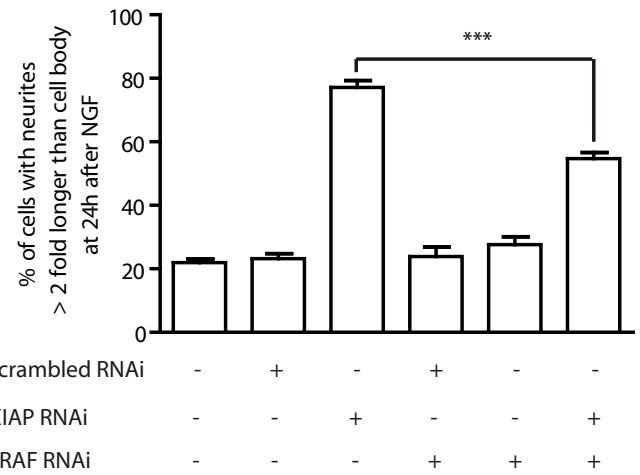

C

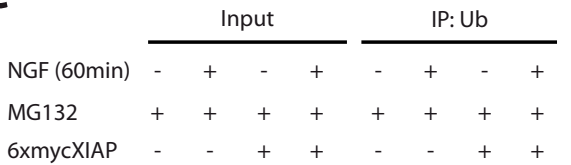

(1.0) (0.6) (1.4) (1.0)

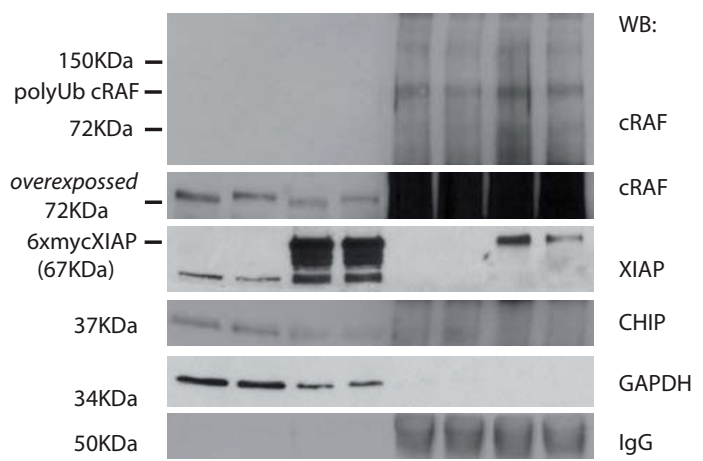

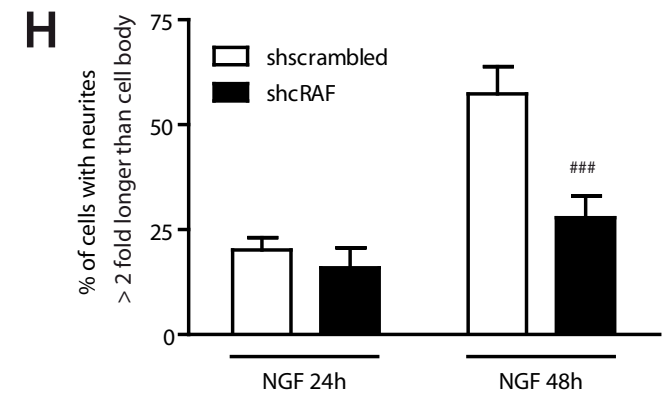

Figure 6 NGF induces cRaf-XIAP dissociationas well as cRaf deubiquitination. (A) NGF-induced cRaf-XIAP dissociation. PC12 cells were treated for $1 \mathrm{hr}$ with NGF and XIAP was immunoprecipitated. cRaf protein levels were detected by immunoblot analysis. cRaf and XIAP protein levels in whole lysate were also detected. (B) cRaf was deubiquitinated by NGF. Cells were serum deprived for 2 hrs and treated with NGF for 1 hr. Proteasome inhibitor MG132 $(10 \mu \mathrm{M})$ or vehicle were present in the medium during starvation and NGF treatment. Ubiquitinated proteins were immunoprecipitated and cRaf was detected by western blot. (C) XIAP overexpression induces cRafubiquitination. Cells were transfected with pcDNA3-6mycXIAP vector and $48 \mathrm{hrs}$ later PC12 cells were treated as in (A). Ubiquitinated proteins were immunoprecipitated and ubiquitination of cRAF was detected by western blot in IP samples (values shown in brackets) or in whole lysates (input). Control without antibody was performed to rule out unspecific binding to Protein $G$ sepharose beads (- Ab). (D) Effect of XIAP up-regulation on cRaf protein levels. PC12 cells were transfected with overexpressing XIAP vectors (pcDNA36 mycXIAP or pBMN-XIAP-I-GFP) or with a control vector (pEGFP). 48 hours after transfection, cells were serum deprived for 6 hours and treated with NGF. cRaf was detected by immunoblot. (E) cRaf protein levels after NGF-stimulation in shXIAP-infected cells. Cells were infected, treated and processed as previously explained. (F) Specific RNA interference for raf1 mRNA was designed and introduced in a lentiviral vector tagged with GFP. Western blot against cRaf was performed to corroborate shRNAi efficiency. $(G)$ Effect of cRaf down regulation on shXIAP-induced neurite outgrowth. Cells were infected with shscrambled and shXIAP alone or with shcRaf. At 3 DIV, cells were serum deprived for 6 hours and treated with NGF in conditioned medium. Results are given as percentage of cells with neurites $>2$ fold longer than cell body and are the mean \pm SEM from 3 independent experiments performed in duplicate. ${ }^{* *} \mathrm{p}<0.001$ vs. shXIAP alone. $(\mathrm{H})$ shcRaf reduces NGF-mediated neurite outgrowth at 48 hours. Cells were infected with shcRaf, serum deprived and treated with NGF. Neurite outgrowth was analysed at 24 and 48 hours after NGF stimulation. \#\#\#p $<0.01$ vs. shscrambled. The gel images were cropped around the region of interest and the samples were resolved on gels run under the same experimental conditions. 
a

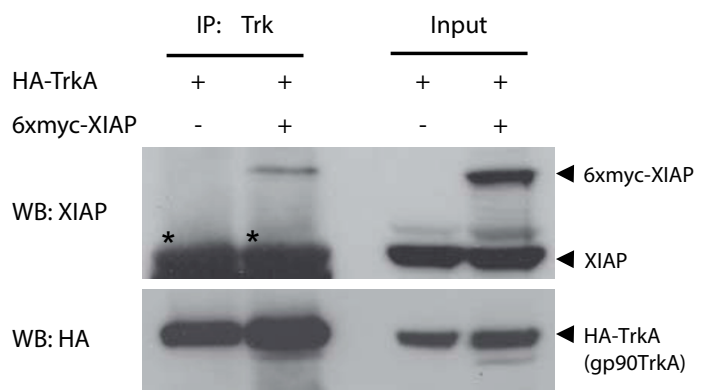

b

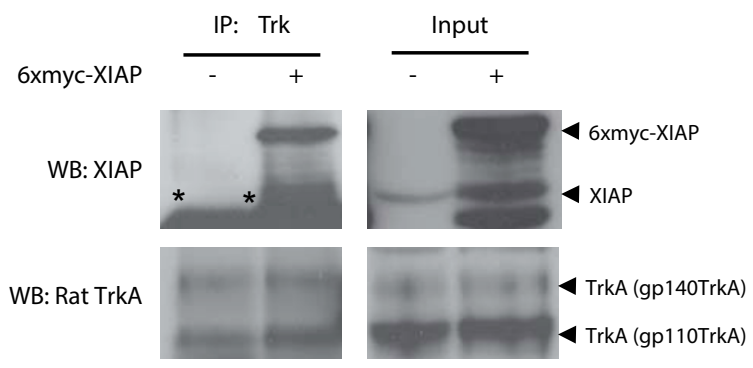

C

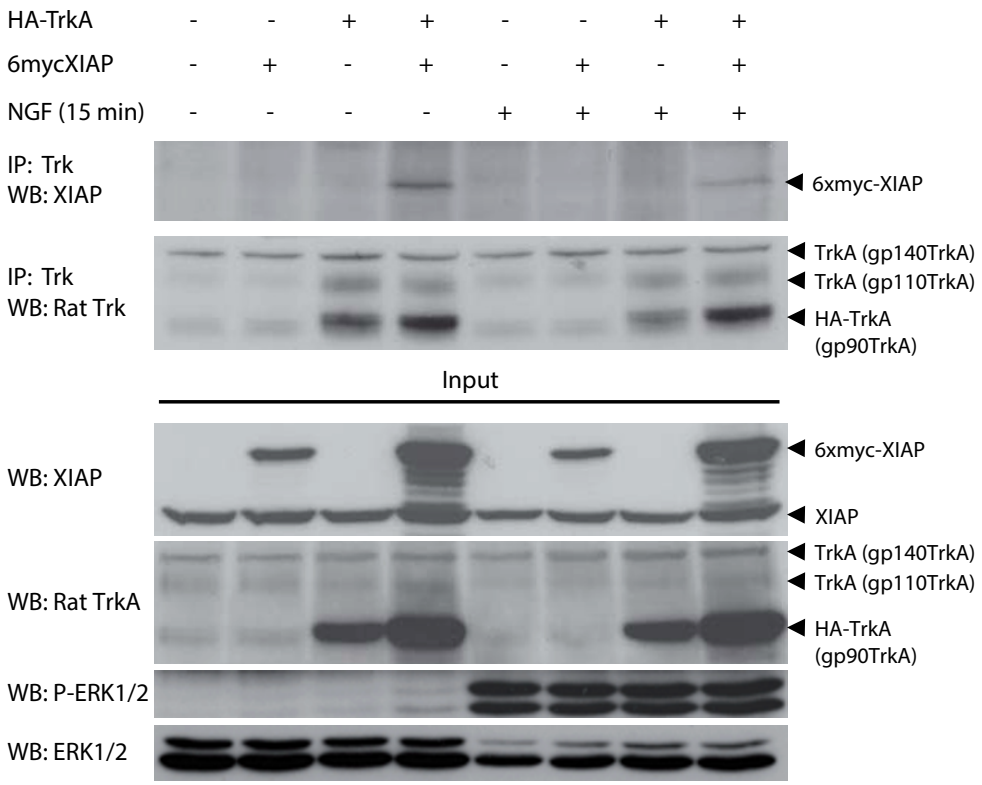

d

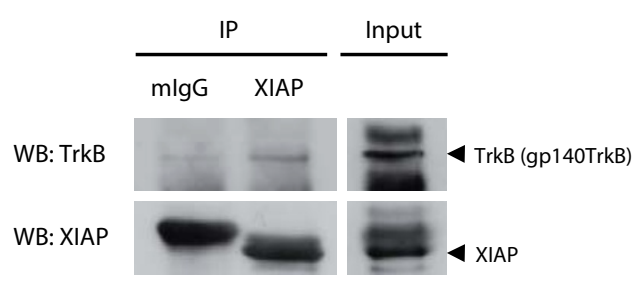

E15 mouse brain lysates

Figure $7 \mid$ XIAP interacts with endogenous Trk in vitro and in vivo. (a) HEK293T cells were transfected with HA-TrkA with or without pcDNA36xmyc-XIAP vector. After 48 hours, Trk was immunoprecipitated using a pan-Trk antibody. HA-tagged Trk and 6xmyc-XIAP as well as endogenous XIAP were detected by western blot using HA and XIAP antibodies respectively. (b) PC12 cells were transfected with pcDNA3-6xmyc-XIAP and endogenous Trk was immunoprecipitated as in (a). The anti-Rat TrkA was used to detect endogenous TrkA with its different glycosylated forms (gp140 and gp110) and anti-XIAP antibody detected both transfected and endogenous XIAP. Asterisks denote the heavy chain immunoglobulins that overlap with endogenous XIAP in both (a) and (b). (c) PC12 cells were transfected during 48 hrs with HA-TrkA, pcDNA3-6xmyc-XIAP or both plasmids and were serum starved for $12 \mathrm{hrs}$ and then stimulated or not with NGF $(100 \mathrm{ng} / \mathrm{mL})$ for 15 minutes. Lysates were subjected to immunoprecipitation using the pan-Trk antibody and immunoblotted with anti-XIAP and anti-Rat TrkA. Inputs were loaded and blotted for XIAP, TrkA, phospho-ERK1/2 and ERK1/2. Note that glycosylation of transfected HA-TrkA is poorer (gp90TrkA) than glycosylation of endogenous trkA (gp140 and gp110). (d) To determine the endogenous interaction of XIAP with TrkB in whole-brain lysates, $2 \mathrm{mg}$ of total protein was immunoprecipitated with anti-XIAP and immunoblotted using anti-TrkB. Rat monoclonal antibody anti-FLAG (mouse IgG) was used as a control. The gel images were cropped around the region of interest and the samples were resolved in the same gel.

structures is closely related to the formation of the axonal growth cone and dendrites ${ }^{31}$. A decrease in XIAP levels was detected in cerebral cortex during development in vivo and in cortical neurons in culture at the time of neuronal arborisation and synaptic maturation. In this context, we decided to explore the eventual relationship between XIAP and neuritogenesis using PC12 cells, a system widely used as standard cell model for neuritogenesis studies.

Several studies have shown that PC12 neuritogenesis is regulated by the neurotrophin NGF. Neurotrophins such as NGF or BDNF not only regulate neuritogenesis in PC12 cells but also in dorsal root ganglia, cortical and hippocampal neurons. NGF induced a reduction in XIAP levels during as soon as $24 \mathrm{hrs}$ post-stimulation, in parallel with the start of neurotrophin-mediated neuritogenesis. The modulatory role of XIAP levels on neuritogenesis in PC12 cells was further confirmed by overexpression of XIAP in PC12 cells, which decreased NGF-mediated neuritogenesis. The regulatory role of XIAP on neuritogenesis was not only observed in PC12 cells but also in primary neuronal cultures in which XIAP down regulation increased dendritic and axonal length.

What is the mechanism underlying XIAP-dependent neuritogenesis regulation? It is well known that NGF-mediated neuritogenesis is dependent on MEK/ERK signalling and accordingly we have observed that pharmacological inhibition of MEK is able to block the neurite outgrowth mediated by shXIAP in NGF-treated PC12 cells. Moreover, down regulation of XIAP increased phospho-ERK levels and the phosphorylation of downstream targets such as CREB. These results indicate that the MEK/ERK pathway mediates the enhancement of NGF-induced neuritogenesis through shXIAP. 
Until now, the only direct link established between XIAP and MEK/ ERK pathway was the interaction with cRaf, which induces the kinase ubiquitination and proteosomal degradation ${ }^{16}$. We have also shown that XIAP and cRaf form a complex in PC12 cells and that this interaction is disrupted in the presence of NGF. Moreover, cRaf ubiquitination is regulated by XIAP and NGF treatment decreases cRaf ubiquitination. However, we were not able to link cRaf ubiquitination toprotein degradation since $c$ Raf levels were not altered in shXIAP infected PC12 cells. Thus, we propose that cRaf ubiquitination and binding to XIAP would regulate its activity, as previously reported with other proteins ${ }^{32}$.

Recent studies have demonstrated that other anti-apoptotic proteins, such as FLIP, are able to support neurotrophin-mediated neurite outgrowth through the regulation of MEK/ERK via their interaction with TrkA or TrkB receptors ${ }^{23}$. Our results also demonstrate, for the first time, that XIAP interacts with TrkA and TrkB receptors. Interaction between TrkA and XIAP is disrupted in the presence of NGF. Binding of NGF to TrkA displaces XIAP from the neurotrophin receptor, facilitating the signalling cascade involved in neuritogenesis. Moreover, binding of XIAP to neurotrophin receptors is not restricted to TrkA since we also report that XIAP binds to TrkB in developing rat brain. Altogether these results support the idea that XIAP could also be a regulator of neurotrophin signalling at the Trk receptors level. Nevertheless, we cannot exclude the possibility that XIAP could also modulate neurite outgrowth by regulating other signalling cascades. For example, the role of $I \kappa \mathrm{B} \alpha$, the NF- $\kappa \mathrm{B}$ inhibitor, in axonal growth and axonal initial segment development ${ }^{33}$ has been described. Also, a recent study demonstrated that XIAP, through its interaction with Rho GDP Dissociation Inhibitor (RhoGDI), is a direct regulator of $\beta$-actin polymerization and cytoskeleton formation ${ }^{34}$.

In summary, our results provide novel evidence of an essential role of XIAP in the formation and elongation of dendrites and axons during neurotrophin-driven neuronal differentiation. Moreover, our report provides new and exciting insights in the molecular mechanisms involved in this novel function of XIAP as a regulator of neurite outgrowth through its interaction with Trk receptors.

\section{Methods}

Cell culture. PC12 cells were grown on culture plates pre-treated with type I collagen $(66.4 \mu \mathrm{g} / \mathrm{ml}$; BD Biosciences) in DMEM supplemented with $7 \%$ heat-inactivated FBS, $7 \%$ heat-inactivated horse serum (Invitrogen), $11.4 \mathrm{mM}$ Hepes, 0.8 units $/ \mathrm{ml}$ penicillin, and $4 \mathrm{mg} / \mathrm{ml}$ streptomycin. HEK293T cells were seed in DMEM supplemented with $10 \%$ heat-inactivated FBS, 4 units $/ \mathrm{ml}$ penicillin, and $20 \mathrm{mg} / \mathrm{ml}$ streptomycin. Cerebrocortical neurons were isolated as described previously ${ }^{35}$. Neurons were initially seeded on culture plates pre-treated with poly-D-lysine $(50 \mu \mathrm{g} / \mathrm{ml})$ in DMEM supplemented with $10 \%$ heat-inactivated FBS, $0.6 \%$ glucose $2 \mathrm{mM}$ L-glutamine, 50 units $/ \mathrm{ml}$ penicillin, and $25 \mathrm{mg} / \mathrm{ml}$ streptomycin. 3 hours after, a medium change was performed with Neurobasal (Gibco) supplemented with B-27 (Gibco), $2 \mathrm{mM}$ L-glutamine, 50 units/ml penicillin, and $25 \mathrm{mg} / \mathrm{ml}$ streptomycin. All the culture plates were maintained at $37^{\circ} \mathrm{C}$ in a humidified $5 \% \mathrm{CO}_{2}$ incubator until the indicate treatments.

Plasmids. Human XIAP cDNA tagged with Myc and expressed under the control of a cytomegalovirus constitutive promoter in the pcDNA3 expression vector and human XIAP expressed in a pBMN vector with an internal ribosomal entry site (IRES) allowing the expression of the green fluorescent protein (GFP) were kindly provided by Dr. RG Korneluk ${ }^{36}$. Enhanced GFP (EGFP) plasmid, used as a transfection control, was obtained from Dr. JM Lizcano (Institut de Neurociencies, UAB, Barcelona, Spain).

For RNA interference (RNAi) experiments, constructs were generated in the pSUPER.retro.puro plasmid (OligoEngine) using specific oligonucleotides against rat XIAP (sequence data available from GeneBank under accession number NM_022231) indicated by capital letter, as follows: RNAi_1, forward, gatcccAGAATCCTATGGTGCAAGAttcaagagaTCTTGCACCATAGGATTCtttttt; reverse, agctaaaaAGAATCCTATGGTGCAAGAtctcttgaaTCTTGCACCATAG GATTCggg; or RNAi_2, forward, gatccccGTAGATAGATGGCAGTATGttcaagaga CATACTGCCATCTATCTACtttttt; reverse, agctaaaaaGTAGATAGATGGCA GTATGtctcttgaaCATACTGCCATCTATCTACggg. A scrambled sequence was used as a control by the following oligonucleotides: RNAi, forward, gatccccAGAACACGA CGGAACAAGAttcaagagaTCTTGTTCCGTCGTGTTCTttttt; reverse,

agctaaaaaAGAACACGACGGAACAAGAtctcttgaaTCTTGTTCCGTCGTGTTCTggg. Construct for cRAF RNA interference experiment was generated using specific oligonucleotides against rat cRAF (sequence data available from GeneBank under accession number NM_012639) as follows: RNAi, forward, gatcccGGGATGAG CTTGCACGACTttcaagagaAGTCGTGCAAGCTCATCCCttttt; and reverse, agctaaaaaGGGATGAGCTTGCACGACTtctcttgaaAGTCGTGCAAGCTCATCCC ggg. Oligonucleotides were provided by Invitrogen and cloned between BglII and HindIII sites of the pSUPER.retro.puro vector, under the control of the DNA Pol III promoter of H1. Lentiviral constructs were achieved by digestion at the EcoRI-ClaI sites to replace $\mathrm{H} 1$ promoter in the lentiviral vector PLVTHM with $\mathrm{H} 1$-short hairpin RNA cassette from pSUPER. The pLVTHM-H1-RNAi was used for lentiviral knockdown experiments.

Lentivirus production. Lentiviruses were propagated as described previously ${ }^{37}$ Briefly, human embryonic kidney cells (HEK293T) cells were seeded at $2.5 \times 10^{5}$ cells $/ \mathrm{ml}$ in dishes with $100 \mathrm{~mm}$ of diameter. At 24 hours, cells were transfected with $20 \mu \mathrm{g}$ of pLVTHM- constructs, $15 \mu \mathrm{g}$ of psPAX2 and $6 \mu \mathrm{g}$ of pMD2.G using the calcium phosphate method. After 48 hours, the medium was centrifuged at $25,000 \mathrm{rpm}$ for $2 \mathrm{~h}$ at $4^{\circ} \mathrm{C}$ and pelleted viruses were resuspended in TNE buffer (50 mMTris- $\mathrm{HCl} \mathrm{pH} \mathrm{7.5,} 130 \mathrm{mMNaCl}$ and $1 \mathrm{mM}$ EDTA) by shaking at $175 \mathrm{rpm}$ overnight at $4{ }^{\circ} \mathrm{C}$. Lentiviruses were stored at $-80^{\circ} \mathrm{C}$. Biological titers of the viral preparations expressed as a number of transducing units (TU) per millilitre were determined by infecting HEK293T cells with serial dilutions. After 48 hours, the percentage of GFP-positive cells was detected by flow cytometry (Cytomics FC 500, Beckman Coulter) and analysed with RXT software.

Cell transfection and infection. Transfection of PC12 cells cultured during 24 hours was performed with the desired plasmids using Lipofectamine 2000 reagent (Invitrogen) following the instructions of the manufacturer. At 2.5 hours after transfection, medium was replaced with the initial culture medium. Lentiviral infection was performed when PC12 or cortical cells were seeded and medium was changed 5 hours after infection. Direct counting of GFP-positive cells monitored the efficiency of infection in each experiment. The percentage of infected cells reached at least $99 \%$ in PC12 cultures and 75\% in cortical cultures. Cell body size did not change under the different conditions in infected cells. The efficiency of both overexpression or down regulation of XIAP was assessed by immunobloting, which demonstrated maximal effects at 48 or 72 hours, respectively.

Neurite measurement. Photographs of random fields of PC12 cells or cortical cultures were taken from two different dishes using an inverted microscope (Nikon eclipse TE200-E). Neurite outgrowth was determined by counting cells with neurites shorter, 1-2 fold longer or more than 2 fold longer than the diameter of the cell body. Double blind analyses were performed by 2 independent observers. Metamorph image analysis software (Molecular Devices) was used for these measurements. In every experiment, more than 100 cells were measured per condition. Measurements from each independent experiment were pooled to represent a distribution of the percentage of cells with neurites falling in the indicated neurite length intervals.

Immunocytochemistry. Infected PC12 (seed at $1.25 \times 10^{4}$ cells $/ \mathrm{ml}$ ) or cortical (seed at $5 \times 10^{4}$ cells $/ \mathrm{ml}$ ) neurons were treated as indicated in 24 -well plates. When required, cellular cultures were washed with ice-cold PBS and fixed with $4 \%$ of paraformaldehyde in PBS for 15 minutes at $4^{\circ} \mathrm{C}$. Afterwards, plates were washed twice with PBS, blocked with $5 \%$ fetal calf serum and $3 \%$ bovine serum albumin (BSA) in TBS (blocking buffer) and then incubated overnight at $4{ }^{\circ} \mathrm{C}$ with specific antibodies detecting Myc ( $1: 100$; Santa Cruz Biotechnology), $\beta$-tubulin $(1: 2,000$; Pharmingen), XIAP (1: 100; BD Biosciences), SMI 312 (1:1,000; Covance) or MAP2 2a $+2 \mathrm{~b}$ $(1: 200$, Sigma). Primary antibodies were visualized using Alexa Fluor-conjugated secondary antibodies (1:500; Molecular Probes) and fluorescence was detected under an inverted microscope (Nikon eclipse TE200-E). Photographs of random fields were taken from different dishes to be analysed. More than 100 cells were measured per condition in each experiment.

Immunoblotting. Cell lysates were cleared by centrifugation at 12,000 rpm for 10 minutes at $4{ }^{\circ} \mathrm{C}$. Protein concentration was quantified by Bradford method. Samples (15-30 $\mu \mathrm{g}$ of protein) were resolved in 10\% SDS-polyacrylamide gels and transferred onto nitrocellulose membranes (Amersham Biosciences). Membranes were washed with TBS with $0.1 \%$ of Tween 20 (TBS-T) and incubated for 1 hour in blocking buffer ( $5 \%$ of non-fat dry milk and $5 \%$ of FBS serum in TBS-T) at room temperature. Blots were then washed andincubated overnight at $4{ }^{\circ} \mathrm{C}$ with primary antibodies against XIAP from BD Biosciences, $\beta$-tubulin (Pharmingen), GAPDH (Ambion), phosphoCREB, phospho-p44/p42 ERK and p44/p42ERK (Cell Signaling), Myc-tag, Histone $\mathrm{H} 1$ and cRaf (Santa Cruz Biotechnology), GFP (Clontech), Ubiquitin (SigmaAldrich), CHIP (Calbiochem), HA (Roche) and TrkA or TrkB (Millipore Corporation). All antibodies were diluted ( $1: 1,000$ for all, except $\beta$-tubulin $1: 2,000$, GAPDH $1: 40,000$ and GFP $1: 50,000)$ in TBS-T with $0.2 \%$ of bovine serum albumin (BSA). Then, blots were washed and incubated for 1 hour at room temperature with specific peroxidise-conjugated secondary antibodies (BD Biosciences) diluted $1: 5,000$ in TBS-T with $5 \%$ of non-fat dry milk. Protein were visualized with the $\mathrm{ECL}^{\mathrm{TM}}$ Western blotting detection reagent (GH Healthcare Europe) and quantified with the Image J software.

Coimmunoprecipitation. PC12 $\left(5 \times 10^{5}\right.$ cells $)$ or HEK293T $\left(3 \times 10^{6}\right.$ cells $)$ transiently transfected or not with human XIAP or/and TrkA were plated in $100 \mathrm{~mm}$ dishes. 3 days after transfection, cells were serum starved for 2 hours and stimulated 
or not with $50 \mathrm{ng} / \mathrm{ml}$ of NGF for 15 or 60 minutes. Cells were collected using lysis buffer and cleared by centrifugation at $12,000 \mathrm{rpm}$. Proteins were quantified by a DC protein assay kit based on the Bradford method (Bio-Rad Laboratories Inc). $1 \mathrm{mg}$ of total protein was taken and adjusted with lysis buffer to achieve a final concentration of $1 \mu \mathrm{g} / \mu \mathrm{l}$. Cell lysates were incubated 1 hour at $4{ }^{\circ} \mathrm{C}$ in an orbital shaker with Protein $\mathrm{G}$ Sepharose (Amersham Biosciences) to discard unspecific binding to beads. Supernatants obtained by centrifugation at $1,000 \mathrm{rpm}$ for 10 minutes were incubated overnight at $4{ }^{\circ} \mathrm{C}$ with specific antibodies for ubiquitin, XIAP orTrk at $1 \mu \mathrm{g} /$ assay. $30 \mu \mathrm{l}$ of Protein G Sepharose beads were added in each sample and incubated 1 hour at $4{ }^{\circ} \mathrm{C}$ in an orbital shaker. Finally, beads were washed three times with lysis buffer, suspended in $30 \mu \mathrm{l}$ of loading buffer containing $1 \mu \mathrm{l}$ of DTT (1 M) and boiled. Samples were loaded onto $10 \%$ SDS-polyacrylamide gel and processed by immunoblotting with the indicated antibodies. Cell lysates (15-30 $\mu$ g protein) blotted with the indicated antibodies were used as input controls. The proteosome inhibitor MG132 $(10 \mu \mathrm{M})$ or vehicle (DMSO; $1: 1000)$ were added in the cell cultures during serum starvation and NGF treatment when ubiquitinated cRAF was monitored. Interaction of TrkB and XIAP was monitor in whole-brain lysates from E15 embryos of C57BI/6 mice obtained after homogenization in lysis buffer with a Polytron homogenizer. As a control, the anti-FLAG-mouse monoclonal antibody was used under the same conditions.

Statistical analysis. All the experiments were performed at least three times. Values were expressed as mean \pm SEM. Student's $t$ test was used to determine the statistical significance of differences in neurite length and branching point numbers $(\mathrm{p}<0.05$ was considered significant). For multiple comparisons, ANOVA was performed followed by Bonferroni's post-test.

1. Hinds, M. G., Norton, R. S., Vaux, D. L. \& Day, C. L. Solution structure of a baculoviral inhibitor of apoptosis (IAP) repeat. Nat Struct Mol Biol 6, 648-651 (1999).

2. Deveraux, Q. L., Takahashi, R., Salvesen, G. S. \& Reed, J. C. X-linked IAP is a direct inhibitor of cell-death proteases. Nature 388, 300-304 (1997).

3. Srinivasula, S. M. \& Ashwell, J. D. IAPs: What's in a Name? Mol Cell 30, 123-135 (2008).

4. Dubrez-Daloz, L., Dupoux, A. \& Cartier, J. IAPS: More than just inhibitors of apoptosis proteins. Cell Cycle 7, 1036-1046 (2008).

5. Varfolomeev, E. et al. c-IAP1 and c-IAP2 Are Critical Mediators of Tumor Necrosis Factor $\alpha(\mathrm{TNF} \alpha)$-induced NF- $\kappa \mathrm{B}$ Activation. J Biol Chem 283, 24295-24299 (2008)

6. Mace, P. D., Shirley, S. \& Day, C. L. Assembling the building blocks: structure and function of inhibitor of apoptosis proteins. Cell Death Differ 17, 46-53 (2009).

7. Riedl, S. J. et al. Structural Basis for the Inhibition of Caspase-3 by XIAP. Cell 104, 791-800 (2001).

8. Chai, J. et al. Structural Basis of Caspase-7 Inhibition by XIAP. Cell 104, 769-780 (2001).

9. Shiozaki, E. N. et al. Mechanism of XIAP-Mediated Inhibition of Caspase-9. Mo Cell 11, 519-527 (2003).

10. Shin, H. et al. Identification of ubiquitination sites on the X-linked inhibitor of apoptosis protein. Biochem. J. 373, 965-971 (2003)

11. Suzuki, Y., Nakabayashi, Y. \& Takahashi, R. Ubiquitin-protein ligase activity of Xlinked inhibitor of apoptosis protein promotes proteasomal degradation of caspase- 3 and enhances its anti-apoptotic effect in Fas-induced cell death. Proc Nat Acad Sci 98, 8662-8667 (2001).

12. Morizane, Y., Honda, R., Fukami, K. \& Yasuda, H. X-Linked Inhibitor of Apoptosis Functions as Ubiquitin Ligase toward Mature Caspase-9 and Cytosolic Smac/DIABLO. J Biochem 137, 125-132 (2005)

13. Singh, J. \& Kaur, G. Neuroprotection mediated by subtoxic dose of NMDA in SHSY5Y neuroblastoma cultures: Activity-dependent regulation of PSA-NCAM expression. Mol Brain Res 137, 223-234 (2005).

14. Winsauer, G., Resch, U., Hofer-Warbinek, R., Schichl, Y. M. \& de Martin, R. XIAP regulates bi-phasic NF- $\mathrm{KB}$ induction involving physical interaction and ubiquitination of MEKK2. Cellular Signalling 20, 2107-2112 (2008).

15. Sobrado, M. et al. Synthesis of Lipoxin A4 by 5-Lipoxygenase Mediates PPAR \{gamma\}-Dependent, Neuroprotective Effects of Rosiglitazone in Experimental Stroke. J Neurosci 29, 3875-3884 (2009).

16. Dogan, T. et al. X-linked and cellular IAPs modulate the stability of C-RAF kinase and cell motility. Nat Cell Biol 10, 1447-1455 (2008).

17. Yamaguchi, K. et al. XIAP, a cellular member of the inhibitor of apoptosis protein family, links the receptors to TAB1-TAK1 in the BMP signaling pathway. EMBO J.18, 179-187 (1999).

18. Gruslin, A., Qiu, Q. \& Tsang, B. K. X-Linked Inhibitor of Apoptosis Protein Expression and the Regulation of Apoptosis During Human Placental Development. Biol Reproduction 64, 1264-1272 (2001).

19. Olayioye, M. A. et al. XIAP-deficiency leads to delayed lobuloalveolar development in the mammary gland. Cell Death Differ 12, 87-90 (2004).

20. Barnes, A. P. \& Polleux, F. Establishment of Axon-Dendrite Polarity in Developing Neurons. Ann Rev Neurosci 32, 347-381 (2009).
21. Liang, Y., Mirnics, Z. K., Yan, C., Nylander, K. D. \& Schor, N. F. Bcl-2 mediates induction of neural differentiation. Oncogene 22, 5515-5518 (2003).

22. Sole, C. et al. The death receptor antagonist FAIM promotes neurite outgrowth by a mechanism that depends on ERK and NF- $\kappa B$ signaling. J.Cell Biol 167, 479-492 (2004).

23. Moubarak, R. S. et al. The Death Receptor Antagonist FLIP-L Interacts with Trk and Is Necessary for Neurite Outgrowth Induced by Neurotrophins. JNeurosci 30, 6094-6105 (2010).

24. Traverse, S., Gomez, N., Paterson, H., Marshall, C. \& Cohen, P. Sustained activation of the mitogen-activated protein (MAP) kinase cascade may be required for differentiation of $\mathrm{PC} 12$ cells. Comparison of the effects of nerve growth factor and epidermal growth factor. Biochem J 288, 351-355 (1992).

25 . Vaudry, D. et al. PACAP protects cerebellar granule neurons against oxidative stress-induced apoptosis. Eur J Neurosci 15, 1451-1460 (2002).

26. Xing, J., Ginty, D. D. \& Greenberg, M. E. Coupling of the RAS-MAPK pathway to gene activation by RSK2, a growth factor-regulated CREB kinase. Science 273, 959-963 (1996).

27. Wixler, V., Smola, U., Schuler, M. \& Rapp, U. Differential regulation of Raf isozymes by growth versus differentiation inducing factors in PC12 pheochromocytoma cells. FEBS Lett 385, 131-137 (1996).

28. Chao, M. V. \& Hempstead, B. L. p75 and Trk: A two-receptor system. Trends Neurosci 18, 321-326 (1995).

29. Galvan, V., Kurakin, A. V. \& Bredesen, D. E. Interaction of checkpoint kinase 1 and the X-linked inhibitor of apoptosis during mitosis. FEBS Letters 558, 57-62 (2004).

30. Zhang, Y. et al. Transfer of siRNA against XIAP induces apoptosis and reduces tumor cells growth potential in human breast cancer "in vitro" and "in vivo". Breast Cancer Res Treat 96, 267-277 (2006).

31. Heiman, M. G. \& Shaham, S. Twigs into branches: how a filopodium becomes a dendrite. Curr Opinion Neurobiol 20, 86-91 (2010).

32. Hurley, J. H. L. S. \& Prag, G. Ubiquitin-binding domains. Biochem J 399, 12 (2006).

33. Sanchez-Ponce, D., Tapia, M., Muñoz, A. \& Garrido, J. J. New role of IKK $\alpha$ / $\beta$ phosphorylated IкB $\alpha$ in axon outgrowth and axon initial segment development. Mol Cell Neurosci 37, 832-844 (2008).

34. Liu, J. et al. X-linked Inhibitor of Apoptosis Protein (XIAP) Mediates Cancer Cell Motility via Rho GDP Dissociation Inhibitor (RhoGDI)-dependent Regulation of the Cytoskeleton. J Biol Chem 286, 15630-15640 (2011)

35. Malagelada, C., Xifro, X., Minano, A., Sabria, J. \& Rodriguez-Alvarez, J. Contribution of caspase-mediated apoptosis to the cell death caused by oxygenglucose deprivation in cortical cell cultures. Neurobiol.Dis. 20, 27-37 (2005).

36. Holcik, M., Lefebvre, C., Yeh, C., Chow, T. \& Korneluk, R. G. A new internalribosome-entry-site motif potentiates XIAP- mediated cytoprotection. Nat Cell Biol 1, 190-192 (1999).

37. Naldini, L. et al. In Vivo Gene Delivery and Stable Transduction of Nondividing Cells by a Lentiviral Vector. Science 272, 263-267 (1996).

\section{Acknowledgments}

This work was supported by grants from Ministerio de Ciencia e Innovación (SAF2011-30281) to J.R.A, SAF2010-19953 to J.X.C. and SAF2010-20925 to C.A.S.), RENEVAS (RD06/0026/1009) to J.R.A, CIBERNED (CB06/05/0042 to J.R.A. and CB06/05/ 1104 to J.X.C.) and Generalitat de Catalunya (SGR2009-1231 to J.R.A. and SGR2009-346 to J.X.C.). R.F. was a recipient of a predoctoral fellowship from the Generalitat de Catalunya and R.M holds a Juan de la Cierva grant from Spanish Government.

\section{Author contributions}

J.R.-A. directed and coordinated the project. R.F., J.X.C. and J.R.-A. conceived and designed the experiments. R.F. and R.S.M. performed experiments. B.B.-Z. and J.M. contributed to the preparation of lentiviral constructs. R.F. and J.V. performed the morphological analyses. A.J.M.-M. contributed to primary cultures transfection. R.F., C.A.S. and J.R.-A. analysed the data. The paper was written by R.F. and J.R.-A. and critically reviewed by all authors.

\section{Additional information}

Supplementary information accompanies this paper at http://www.nature.com/ scientificreports

Competing financial interests: The authors declare no competing financial interests. How to cite this article: Fadó, R. et al. X-linked Inhibitor of Apoptosis Protein negatively regulates neuronal differentiation through interaction with cRAF and Trk. Sci. Rep. 3, 2397; DOI:10.1038/srep02397 (2013).

This work is licensed under a Creative Commons AttributionNonCommercial-NoDerivs 3.0 Unported license. To view a copy of this license, visit http://creativecommons.org/licenses/by-nc-nd/3.0 